\title{
SOLVABILITY OF A CLASS OF BRAIDED FUSION CATEGORIES
}

\author{
SONIA NATALE AND JULIA YAEL PLAVNIK
}

\begin{abstract}
We show that a weakly integral braided fusion category $\mathcal{C}$ such that every simple object of $\mathcal{C}$ has Frobenius-Perron dimension $\leq 2$ is solvable. In addition, we prove that such a fusion category is grouptheoretical in the extreme case where the universal grading group of $\mathcal{C}$ is trivial.
\end{abstract}

\section{INTRODUCTION AND MAIN RESULTS}

Let $k$ be an algebraically closed field of characteristic zero. A fusion category over $k$ is a semisimple tensor category over $k$ having finitely many isomorphism classes of simple objects. In this paper we consider the problem of giving structural results of a fusion category $\mathcal{C}$ under restrictions on the set c.d.(C) of Frobenius-Perron dimensions of its simple objects.

Results of this type were obtained in the paper [20. For instance, we showed in [20, Theorem 7.3] that under the assumption that $\mathcal{C}$ is braided odd-dimensional and c. d. $(\mathcal{C}) \subseteq\left\{p^{m}: m \geq 0\right\}$, where $p$ is a (necessarily odd) prime number, then $\mathcal{C}$ is solvable. Also, the same is true when $\mathcal{C}=\operatorname{Rep} H$, where $H$ is a semisimple quasitriangular Hopf algebra and c.d. $(\mathcal{C})=\{1,2\}$ [20, Theorem 6.12].

Using results of the paper [1], we also showed in [20, Theorem 6.4] that if $\mathcal{C}=\operatorname{Rep} H$, where $H$ is any semisimple Hopf algebra, and c. d. $(\mathcal{C}) \subseteq\{1,2\}$, then $\mathcal{C}$ is weakly group-theoretical, and furthermore, it is group-theoretical if $\mathcal{C}$ coincides with the adjoint subcategory $\mathcal{C}_{\text {ad }}$.

Our main results are the following theorems. Recall that a fusion category $\mathcal{C}$ is called weakly integral if the Frobenius-Perron dimension of $\mathcal{C}$ is a natural integer.

Theorem 1.1. Let $\mathcal{C}$ be a weakly integral braided fusion category such that FPdim $X \leq 2$, for all simple object $X$ of $\mathcal{C}$. Then $\mathcal{C}$ is solvable.

Theorem 1.1 extends the previous result for semisimple quasitriangular Hopf algebras mentioned above. It implies in particular that every weakly integral braided fusion category with Frobenius-Perron dimensions of simple

Date: May 10, 2012.

1991 Mathematics Subject Classification. 18D10; $16 \mathrm{~T} 05$.

Key words and phrases. Fusion category; braided fusion category; solvability.

The research of S. N. was partially supported by CONICET and Secyt-UNC. The research of J. P. was partially supported by CONICET, ANPCyT and Secyt-UNC. 
objects at most 2 is weakly group-theoretical. This gives some further support to the conjecture that every weakly integral fusion category is weakly group-theoretical. See [8, Question 2].

It is known that a nilpotent braided fusion category, which is in addition integral (that is, c.d. $(\mathcal{C}) \subseteq \mathbb{Z}_{+}$) is always group-theoretical [4, Theorem 6.10]. We also show that the same conclusion is true in the opposite extreme case:

Theorem 1.2. Let $\mathcal{C}$ be a weakly integral braided fusion category such that FPdim $X \leq 2$, for all simple object $X$ of $\mathcal{C}$. Suppose that the universal grading group of $\mathcal{C}$ is trivial. Then $\mathcal{C}$ is group-theoretical.

Theorems 1.1 and 1.2 are proved in Section 4, Our proofs rely on the results of Naidu and Rowell [18] for the case where $\mathcal{C}$ is integral and has a faithful self-dual simple object of Frobenius-Perron dimension 2.

Being group-theoretical, a braided fusion category $\mathcal{C}$ satisfying the assumptions of Theorem 1.2, has the so called property $\mathbf{F}$, namely, all associated braid group representations on the tensor powers of objects of $\mathcal{C}$ factor over finite groups. See [9, Corollary 4.4]. It is conjectured that every braided weakly integral fusion category does have property $\mathbf{F}[18$. This conjecture has been proved for braided fusion categories $\mathcal{C}$ with c. d. $(\mathcal{C})=\{1,2\}$ such that all objects of $\mathcal{C}$ are self-dual or $\mathcal{C}$ is generated by a self-dual simple object [18, Corollary 4.3 and Remark 4.4].

The paper is organized as follows. In Section 2 we recall the main facts and terminology about fusion and braided fusion categories used throughout. In Section 3 we discuss some families of (integral) examples that appear in the literature. We also recall in this section the results of the paper [18] related to dihedral group fusion rules that will be used later. In Section 4 we give the proofs of Theorems 1.1 and 1.2 .

\section{Preliminaries}

2.1. Fusion categories. Let $\mathcal{C}$ be a fusion category. We shall denote by $\operatorname{Irr}(\mathcal{C})$ the set of isomorphism classes of simple objects of $\mathcal{C}$ and by $G(\mathcal{C})$ the group of isomorphism classes of invertible objects of $\mathcal{C}$. For an object $X$ of $\mathcal{C}$, we shall indicate by $\mathcal{C}[X]$ the fusion subcategory generated by $X$ and by $G[X]$ the subgroup of $G(\mathcal{C})$ consisting of invertible objects $g$ such that $g \otimes X \simeq X$.

If $\mathcal{D}$ is another fusion category, $\mathcal{C}$ and $\mathcal{D}$ are Morita equivalent if $\mathcal{D}$ is equivalent to the dual $\mathcal{C}_{\mathcal{M}}^{*}$ with respect to an indecomposable module category $\mathcal{M}$. Recall that $\mathcal{C}$ is called pointed if all its simple objects are invertible and it is called group-theoretical if it is Morita equivalent to a pointed fusion category.

There is a canonical faithful grading $\mathcal{C}=\oplus_{g \in U(\mathcal{C})} \mathcal{C}_{g}$, with trivial component $\mathcal{C}_{e}=\mathcal{C}_{\text {ad }}$, where $\mathcal{C}_{\text {ad }}$ is the adjoint subcategory of $\mathcal{C}$, that is, the fusion subcategory generated by $X \otimes X^{*}$, where $X$ runs through the simple objects of $\mathcal{C}$. The group $U(\mathcal{C})$ is called the universal grading group of $\mathcal{C}$. $\mathcal{C}$ is called 
nilpotent if the upper central series $\cdots \subseteq \mathcal{C}^{(n+1)} \subseteq \mathcal{C}^{(n)} \subseteq \cdots \subseteq \mathcal{C}^{(0)}=\mathcal{C}$ converges to $\operatorname{Vec}_{k}$, where $\mathcal{C}^{(i)}:=\left(\mathcal{C}^{(i-1)}\right)_{\text {ad }}, i \geq 1$. See [11].

A weakly group-theoretical fusion category is a fusion category $\mathcal{C}$ which is Morita equivalent to a nilpotent fusion category. If $\mathcal{C}$ is Morita equivalent to a cyclically nilpotent fusion category, then $\mathcal{C}$ is called solvable. We refer the reader to [7, 8] for further definitions and facts about fusion categories.

2.2. Braided fusion categories. Let $\mathcal{C}$ be a braided fusion category, that is, $\mathcal{C}$ is equipped with natural isomorphisms $c_{X, Y}: X \otimes Y \rightarrow Y \otimes X, X, Y \in \mathcal{C}$, satisfying the hexagon axioms. Recall that $\mathcal{C}$ is called premodular if it is also spherical, that is, $\mathcal{C}$ has a pivotal structure such that left and right categorical dimensions coincide. Equivalently, $\mathcal{C}$ is premodular if it is endowed with a compatible ribbon structure [2, 16].

We say that the objects $X$ and $Y$ of a braided fusion category $\mathcal{C}$ centralize each other if $c_{Y, X} c_{X, Y}=\mathrm{id}_{X \otimes Y}$. The centralizer $\mathcal{D}^{\prime}$ of a fusion subcategory $\mathcal{D} \subseteq \mathcal{C}$ is defined to be the full subcategory of objects of $\mathcal{C}$ that centralize every object of $\mathcal{D}$. The centralizer $\mathcal{D}^{\prime}$ results a fusion subcategory of $\mathcal{C}$.

The Müger (or symmetric) center $Z_{2}(\mathcal{C})$ of $\mathcal{C}$ is $Z_{2}(\mathcal{C})=\mathcal{C}^{\prime}$; this is a symmetric fusion subcategory of $\mathcal{C}$ whose objects are called central, degenerate or transparent. A braided fusion category $\mathcal{C}$ is called non-degenerate if its Müger center $Z_{2}(\mathcal{C})$ is trivial. A modular category is a non-degenerate premodular category $\mathcal{C}$.

Remark 2.1. Recall that a fusion category $\mathcal{C}$ is called pseudo-unitary if $\operatorname{dim} \mathcal{C}=\mathrm{FP} \operatorname{dim} \mathcal{C}$, where $\operatorname{dim} \mathcal{C}$ is the global dimension of $\mathcal{C}$ and $\mathrm{FPdim} \mathcal{C}$ is the Frobenius-Perron dimension of $\mathcal{C}$. If $\mathcal{C}$ pseudo-unitary then $\mathcal{C}$ has a canonical spherical structure with respect to which categorical dimensions of all simple objects coincide with their Frobenius-Perron dimensions [7, Proposition 8.23].

In particular, this holds for any weakly integral fusion category, because it is automatically pseudo-unitary [7, Proposition 8.24]. Hence every weakly integral non-degenerate fusion category is canonically a modular category.

\section{SOME FAMILIES OF EXAMPLES}

\subsection{Examples of fusion categories with Frobenius-Perron dimen-} sions $\leq 2$. In this subsection we discuss examples of weakly integral fusion categories with Frobenius-Perron dimensions of simple objects $\leq 2$ that appear in the literature.

Example 3.1. Consider a Hopf algebra $H$ fitting into an abelian exact sequence:

$$
k \rightarrow k^{\Gamma} \rightarrow H \rightarrow k \mathbb{Z}_{2} \rightarrow k,
$$

where $\Gamma$ is a finite group. Let $\mathcal{C}=\operatorname{Rep} H$. Then c. d. $(\mathcal{C}) \subseteq\{1,2\}$ and equality holds if the associated action of $\mathbb{Z}_{2}$ on $\Gamma$ is not trivial. 
All these examples are group-theoretical, in view of [19, Theorem 1.3]. Observe that, as a consequence of [1, Theorem 6.4], any cosemisimple Hopf algebra $H$ such that c. d. $(\mathcal{C}) \subseteq\{1,2\}$ is group-theoretical if $\mathcal{C}=\mathcal{C}_{\text {ad }}$. See [20, Theorem 6.4].

Non-trivial examples of cosemisimple Hopf algebras fitting into an exact sequence (3.1) are given by the Hopf algebras

$$
\mathcal{A}_{4 m}^{*}, \mathcal{B}_{4 m}^{*} \quad m \geq 2
$$

of dimension $4 m$, due to Masuoka [14]. In these cases, $\Gamma$ is a dihedral group.

Example 3.2. Let $\mathcal{C}=\mathcal{T} \mathcal{Y}(G, \chi, \tau)$ be the Tambara-Yamagami category associated to a finite (necessarily abelian) group $G$, a symmetric non-degenerate bicharacter $\chi: G \times G \rightarrow k^{\times}$and an element $\tau \in k$ satisfying $|G| \tau^{2}=1$ [24]. This is a fusion category with isomorphism classes of simple objects parameterized by the set $G \cup\{X\}$, where $X \notin G$, obeying the fusion rules

$$
g \otimes h=g h, \quad g, h \in G, \quad X \otimes X=\oplus_{g \in G} g .
$$

We have c. d. $(\mathcal{C})=\{1,2\}$ if and only if $G$ is of order 4 . Therefore, in this case $\mathrm{FP} \operatorname{dim} \mathcal{C}=8$.

If $G \simeq \mathbb{Z}_{4}$, there are two possible fusion categories $\mathcal{C}$. None of them is braided [22, Theorem $1.2(1)]$.

If $G \simeq \mathbb{Z}_{2} \times \mathbb{Z}_{2}$ there are exactly four classes of Tambara-Yamagami categories with irreducibles degrees 1 or 2 , by [24, Theorem 4.1]. Three of them are (equivalent to) the categories of representations of eight-dimensional Hopf algebras: the dihedral group algebra of order 8, the quaternion group algebra, and the Kac-Paljutkin Hopf algebra $H_{8}$. The remaining fusion category, which has the same $\chi$ as $H_{8}$ but $\tau=-1 / 2$, is not realized as the fusion category of representations of a Hopf algebra. Since in this case $G$ is an elementary abelian 2-group all of this categories admit a braiding, by [22, Theorem $1.2(1)$.

All the fusion categories in this example are group-theoretical. In fact, by [10, Lemma 4.5], for any symmetric non-degenerate bicharacter $\chi: G \times$ $G \rightarrow k^{\times}, G$ contains a Lagrangian subgroup with respect to $\chi$. Therefore $\mathcal{T Y}(G, \chi, \tau)$ is group-theoretical, by [10, Theorem 4.6].

Example 3.3. Recall that a near-group category is a fusion category with exactly one isomorphism class of non-invertible simple object. In the notation of [22], the fusion rules of $\mathcal{C}$ are determined by a pair $(G, \kappa)$, where $G$ is the group of invertible objects of $\mathcal{C}$ and $\kappa$ is a nonnegative integer. Letting $\operatorname{Irr}(\mathcal{C})=G \cup\{X\}$, where $X$ is non-invertible, we have the relation

$$
X \otimes X=\oplus_{g \in G} g \oplus \kappa X .
$$

Near-group categories with fusion rule $(G, 0)$ for some finite group $G$ are thus Tambara-Yamagami categories, discussed in the previous example. Let us consider near-group categories with fusion rule $(G, \kappa)$ for some finite group $G$ and a positive integer $\kappa$. 
We have c.d.(C) $=\{1,2\}$ if and only if $G$ is of order 2 and $\kappa=1$, that means $\mathcal{C}$ is of type $\left(\mathbb{Z}_{2}, 1\right)$. Therefore, in this case FPdim $\mathcal{C}=6$ and since $\kappa>0$, then $\mathcal{C}$ is group-theoretical, by [6, Theorem 1.1]. By [25, Theorem $1.5]$, there are up to equivalence exactly two non-symmetric braided neargroup categories with fusion rule $\left(\mathbb{Z}_{2}, 1\right)$.

Example 3.4. Examples of a weakly integral braided fusion categories which are not integral and Frobenius-Perron dimensions of simple objects are $\leq 2$ are given by the Ising categories, studied in [5, Appendix B]. In this case, there is a unique non-invertible simple object $X$ with $X^{\otimes 2}=\mathbf{1} \oplus a$, where $a$ generates the group of invertible objects, isomorphic to $\mathbb{Z}_{2}$ (note that these are also Tambara-Yamagami categories). We have here c. d. $(\mathcal{C})=\{1, \sqrt{2}\}$ and $\mathrm{FPdim} \mathcal{C}=4$. Every braided Ising category is modular [5, Corollary B.12].

Other examples come from braided fusion categories with generalized Tambara-Yamagami fusion rules of type $\left(G, \mathbb{Z}_{2}\right)$, where $G$ is a finite group. See [13. In these examples, $\mathcal{C}$ is not pointed, the group of invertible objects is $G$, and $\mathbb{Z}_{2} \simeq \Gamma \subseteq G$ is a subgroup such that $X \otimes X^{*} \simeq \oplus_{h \in \Gamma} h$, for all non-invertible object $X$ of $\mathcal{C}$. Hence we also have c. d. $(\mathcal{C})=\{1, \sqrt{2}\}$.

Since they are not integral, these examples are not group-theoretical.

Example 3.5. Let $\mathcal{C}$ be a braided group-theoretical fusion category. Then $\mathcal{C}$ is an equivariantization of a pointed fusion category, that is, $\mathcal{C} \simeq \mathcal{D}^{G}$, where $\mathcal{D}$ is a pointed fusion category and $G$ is a finite group acting on $\mathcal{D}$ by tensor autoequivalences [17]. In this case, $\mathcal{C}$ contains the category $\operatorname{Rep} G$ of finite-dimensional representations of $G$ as a fusion subcategory.

Suppose that c.d. $(\mathcal{C})=\{1, p\}$, where $p$ is any prime number. Then also c. d. $(G) \subseteq\{1, p\}$. In particular, the group $G$ must have a normal abelian p-complement; moreover, either $G$ contains an abelian normal subgroup of index $p$ or the center $Z(G)$ has index $p^{3}$. See [12, Theorems 6.9, 12.11].

3.2. Fusion rules of dihedral type. Let $D_{n}$ be the dihedral group of order $2 n, n \geq 1$. Recall that $D_{n}$ has a presentation by generators $t, z$ and relations $t^{2}=1=z^{n}, t z=z^{-1} t$.

The following proposition describes the fusion rules of $\operatorname{Rep} D_{n}$ (c.f. [14]).

Proposition 3.6. (1) Suppose $n$ is odd. Then the isomorphisms classes of simple objects of Rep $D_{n}$ are represented by 2 invertible objects, 1 and $g$, and $r=(n-1) / 2$ simple objects $X_{1}, \ldots, X_{r}$, of dimension 2, such that

$$
\begin{aligned}
& g \otimes X_{i}=X_{i}=X_{i} \otimes g, \quad \forall i=1, \ldots, r \\
& X_{i} \otimes X_{j}= \begin{cases}X_{i+j} \oplus X_{|i-j|}, & \text { if } i+j \leq r, \\
X_{n-(i+j)} \oplus X_{|i-j|}, & \text { if } i+j>r\end{cases}
\end{aligned}
$$

where $X_{0}=1 \oplus g$.

(2) Suppose $n$ is even, that is $n=2 m$. Then the isomorphisms classes of simple objects of $\operatorname{Rep} D_{n}$ are represented by 4 invertible objects, 1 , 
$g, h, f=g h$, and $m-1$ simple objects $X_{1}, \ldots, X_{m-1}$, of dimension 2 , such that

$$
\begin{aligned}
& g \otimes X_{i}=X_{i}=X_{i} \otimes g, \quad \forall i=1, \ldots, m-1, \\
& h \otimes X_{i}=X_{m-i}=X_{i} \otimes h, \quad \forall i=1, \ldots, m-1, \\
& X_{i} \otimes X_{j}= \begin{cases}X_{i+j} \oplus X_{|i-j|}, & \text { if } i+j \leq m, \\
X_{2 m-(i+j)} \oplus X_{|i+j|}, & \text { if } i+j>m ;\end{cases}
\end{aligned}
$$

where $X_{0}=1 \oplus g$ and $X_{m}=h \oplus f$.

In particular, the group of invertible objects in $\operatorname{Rep} D_{n}$ is isomorphic to $\mathbb{Z}_{2}$ if $n$ is odd, and to $\mathbb{Z}_{2} \times \mathbb{Z}_{2}$ if $n$ is even.

Remark 3.7. Suppose that 4 divides $n=2 m$. Then $X_{m / 2}$ is fixed under (left and right) multiplication by all invertible objects of $\operatorname{Rep} D_{n}$.

Let $\mathcal{C}$ be a fusion category with c.d. $(\mathcal{C})=\{1,2\}$. Suppose that the Grothendieck ring of $\mathcal{C}$ is commutative (for example, this is the case if $\mathcal{C}$ is braided). Assume in addition that the following conditions hold:

(a) All objects are self-dual, that is $X \simeq X^{*}$, for every object $X$ of $\mathcal{C}$.

(b) $\mathcal{C}$ has a faithful simple object.

Then, it is shown in [18, Theorem 4.2] that $\mathcal{C}$ is Grothendieck equivalent to $\operatorname{Rep} D_{n}$. Moreover, $\mathcal{C}$ is necessarily group-theoretical.

It is possible to remove the assumption that all the objects are self-dual but it is still necessary the condition of self-duality on the faithful simple object. Namely, suppose that $\mathcal{C}$ is not self-dual, but satisfies

(b') $\mathcal{C}$ has a faithful self-dual simple object.

In this case $\mathcal{C}$ is still group-theoretical and it is Grothendieck equivalent to Rep $\widetilde{D}_{n}, n$ odd. See [18, Remark 4.4]. Here $\widetilde{D}_{n}$ is the generalized quaternion (binary dihedral) group of order $4 n$, that is, the group presented by generators $a, s$, with relations $a^{2 n}=1, s^{2}=a^{n}, s^{-1} a s=a^{-1}$. (Observe that for $n$ odd, $\widetilde{D}_{n}$ is isomorphic to the semidirect product $\mathbb{Z}_{n} \rtimes \mathbb{Z}_{4}$, with respect to the action given by inversion, considered in [18]. For even $n$, Rep $\widetilde{D}_{n}$ is Grothendieck equivalent to $\operatorname{Rep} D_{2 n}$, while $\mathbb{Z}_{n} \rtimes \mathbb{Z}_{4}$ has no faithful representation of degree 2.)

Lemma 3.8. Let $n \geq 2$. Then $\left(\operatorname{Rep} \widetilde{D}_{n}\right)_{\mathrm{ad}}=\operatorname{Rep} D_{n}$. In addition,

$$
\left(\operatorname{Rep} D_{n}\right)_{\mathrm{ad}}= \begin{cases}\operatorname{Rep} D_{n / 2}, & \text { if } n \text { is even, } \\ \operatorname{Rep} D_{n}, & \text { if } n \text { is odd. }\end{cases}
$$

Proof. Recall that when $\mathcal{C}=\operatorname{Rep} G$, where $G$ is a finite group, then $\mathcal{C}_{\text {ad }}=$ $\operatorname{Rep} G / Z(G)$ [11. The first claim follows from the fact that the center of $\tilde{D}_{n}$ equals $\left\{1, s^{2}\right\} \simeq \mathbb{Z}_{2}$. On the other hand, the center $Z\left(D_{n}\right)$ is trivial if $n$ is odd, and equals $\left\{1, z^{n / 2}\right\} \simeq \mathbb{Z}_{2}$ if $n$ is even. This implies the second claim and finishes the proof of the lemma. 


\section{Proof of the MAIN RESUlts}

In this section we shall prove Theorems 1.1 and 1.2 .

Proposition 4.1. Let $\mathcal{C}$ be a premodular fusion category. Suppose $\mathcal{C}$ has an invertible object $g$ of order $n$ and a simple object $X$ such that

(4.1) $g \otimes X=X$, and

(4.2) $g$ centralizes $X$.

Then we have

(i) $\mathcal{C}$ is an equivariantization by the cyclic group $\mathbb{Z}_{n}$ of a fusion category $\widetilde{\mathcal{C}}$.

(ii) If $g \in \mathcal{C}^{\prime}$, then $\widetilde{\mathcal{C}}$ is braided.

Proof. Condition (4.1) ensures the existence of a fiber functor on the fusion category $\mathcal{C}[g]$ generated by $g$. Then $\mathcal{C}[g]$ is equivalent to $\operatorname{Rep} \mathbb{Z}_{n}$ as fusion categories.

Moreover, they are equivalent as braided fusion categories. Indeed, (4.1) implies $\mathcal{C}[g] \subseteq \mathcal{C}[X]$ and therefore $\mathcal{C}[g] \subseteq Z_{2}(\mathcal{C}[X])$, by (4.2). Hence $\mathcal{C}[g]$ is symmetric. Then the only possible twists in $\mathcal{C}$ are $\theta_{h}=1$ and $\theta_{h}=-1$ for all $h \in\langle g\rangle$. But $\theta_{h}$ is not equal to -1 since $h$ centralizes $X$ and $h \otimes X=X$ [15. Lemma 5.4]. Then $\theta_{h}=1$ for all $h \in\langle g\rangle$. Therefore $\mathcal{C}[g] \simeq \operatorname{Rep} \mathbb{Z}_{n}$ as braided fusion categories, as claimed.

Let $\Gamma=\langle g\rangle \subseteq G(\mathcal{C})$. It follows from [5, Theorem 4.18 (i)] that the deequivariantization $\widetilde{\mathcal{C}}=\mathcal{C}_{\Gamma}$ of $\mathcal{C}$ by $\Gamma$ is a fusion category and there is a canonical equivalence $\mathcal{C} \simeq \widetilde{\mathcal{C}}^{\Gamma}$ between the category $\mathcal{C}$ and the $\Gamma$-equivariantization of $\widetilde{\mathcal{C}}$, which shows (i).

Furthermore, if $g \in \mathcal{C}^{\prime}$ then $\widetilde{\mathcal{C}}$ is braided and the equivalence $\mathcal{C} \simeq \widetilde{\mathcal{C}}^{\Gamma}$ is of braided fusion categories [2, 15] (see also [5, Theorem 4.18 (ii)]). Thus we get (ii). This proves the proposition.

Lemma 4.2. Let $\mathcal{C}$ be a fusion category with commutative Grothendieck ring. Suppose that $\mathcal{C}=\mathcal{C}_{\mathrm{ad}}$. If $\mathcal{D}_{1}, \ldots, \mathcal{D}_{s}$ are fusion subcategories that generate $\mathcal{C}$ as a fusion category, then $\mathcal{D}_{1}^{(m)}, \ldots, \mathcal{D}_{s}^{(m)}$ generate $\mathcal{C}$ as a fusion category, $\forall m \geq 0$.

Proof. Since $\mathcal{D}_{1}, \ldots, \mathcal{D}_{s}$ generate $\mathcal{C}$, then $\left(\mathcal{D}_{1}\right)_{\text {ad }}, \ldots,\left(\mathcal{D}_{s}\right)_{\text {ad }}$ generate $\mathcal{C}$. In fact, let $X$ be a simple object of $\mathcal{C}$. There exist simple objects $X_{i_{1}}, \ldots, X_{i_{t}}$, with $X_{i_{l}} \in \mathcal{D}_{i_{l}}, 1 \leq i_{1}, \ldots, i_{t} \leq s$, such that $X$ is a direct summand of $X_{i_{1}} \otimes \ldots \otimes X_{i_{t}}$. Then $X \otimes X^{*}$ is a direct summand of

$$
X_{i_{1}} \otimes \ldots \otimes X_{i_{t}} \otimes X_{i_{t}}^{*} \otimes \ldots \otimes X_{i_{1}}^{*} \simeq\left(X_{i_{1}} \otimes X_{i_{1}}^{*}\right) \otimes \ldots \otimes\left(X_{i_{t}} \otimes X_{i_{t}}^{*}\right),
$$

where we have used that $\mathcal{C}$ has a commutative Grothendieck ring. Notice that the object in the right hand side belongs to the fusion subcategory generated by $\left(\mathcal{D}_{1}\right)_{\text {ad }}, \ldots,\left(\mathcal{D}_{s}\right)_{\text {ad }}$. Since $X$ was arbitrary, it follows that $\left(\mathcal{D}_{1}\right)_{\text {ad }}, \ldots,\left(\mathcal{D}_{s}\right)_{\text {ad }}$ generate $\mathcal{C}_{\text {ad }}$. But $\mathcal{C}=\mathcal{C}_{\text {ad }}$ by assumption, then we have 
proved that $\left(\mathcal{D}_{1}\right)_{\text {ad }}, \ldots,\left(\mathcal{D}_{s}\right)_{\text {ad }}$ generate $\mathcal{C}$. The statement follows from this by induction on $n$, since $\mathcal{D}_{j}^{(n)}=\left(\mathcal{D}_{j}^{(n-1)}\right)_{\text {ad }}$, for all $j=1, \ldots s, n \geq 1$.

4.1. Braided fusion categories with irreducible degrees 1 and 2 . Throughout this subsection $\mathcal{C}$ is a braided fusion category with c. d. $(\mathcal{C})=$ $\{1,2\}$. We regard $\mathcal{C}$ as a premodular category with respect to its canonical spherical structure. See Remark 2.1.

Remark 4.3. Note that $G[X] \neq \mathbf{1}$, for all $X$ such that FPdim $X=2$. Moreover, $|G[X]|=2$ or 4 . In particular the (abelian) group $G(\mathcal{C})$ is not trivial.

Proposition 4.4. Let $g$ be a non-trivial invertible object such that $g^{2}=1$ and $\theta_{g}=1$. Assume that $g$ generates the Müger center $\mathcal{C}^{\prime}$ of $\mathcal{C}$ as a fusion category. Then $\mathcal{C}$ is the equivariantization of a modular fusion category $\widetilde{\mathcal{C}}$ by the group $\mathbb{Z}_{2}$. Furthermore c. d. $(\widetilde{\mathcal{C}}) \subseteq\{1,2\}$.

Proof. By assumption $\mathcal{C}^{\prime} \simeq \operatorname{Rep} \mathbb{Z}_{2}$ is tannakian. Then the de-equivariantization $\widetilde{\mathcal{C}}$ of $\mathcal{C}$ by $\mathcal{C}^{\prime}$ is a modular category and there is an action of $\mathbb{Z}_{2}$ on $\widetilde{\mathcal{C}}$ such that $\mathcal{C} \simeq \widetilde{\mathcal{C}}^{\mathbb{Z}_{2}}$ [2, 15]. Since c.d. $\left(\widetilde{\mathcal{C}}^{\mathbb{Z}_{2}}\right)=$ c.d. $(\mathcal{C})=\{1,2\}$, then c. d. $(\widetilde{\mathcal{C}}) \subseteq\{1,2\}$, by [8, Proof of Proposition 6.2], [20, Lemma 7.2].

Lemma 4.5. Suppose that $\mathcal{C} \neq \mathcal{C}_{\mathrm{ad}}$ and $\mathcal{C}_{\mathrm{ad}}$ is solvable. Then $\mathcal{C}$ is solvable.

Proof. Since $\mathcal{C}$ is braided, its universal grading group $U(\mathcal{C})$ is abelian 11, Theorem 6.2]. The category $\mathcal{C}$ is a $U(\mathcal{C})$-extension of $\mathcal{C}_{\text {ad }}$ and an extension of a solvable category by a solvable group is again solvable [ 8 , Proposition 4.5 (i)]. Then $\mathcal{C}$ is solvable, as claimed.

Lemma 4.6. Assume $\mathcal{C}=\mathcal{C}_{\text {ad }}$. Then $\operatorname{FPdim} \mathcal{C}^{\prime} \geq 2$.

Proof. Suppose on the contrary that $\mathrm{FPdim} \mathcal{C}^{\prime}=1$, that is, $\mathcal{C}$ is modular. Then, by [11, Theorem 6.2], $U(\mathcal{C}) \simeq \widehat{G(\mathcal{C})} \simeq G(\mathcal{C})$. By Remark 4.3, $\mathcal{C}_{\text {ad }} \subsetneq \mathcal{C}$, against the assumption. Hence $\mathrm{FPdim} \mathcal{C}^{\prime} \geq 2$, as claimed.

Lemma 4.7. Suppose $\mathcal{C}$ is generated by a simple object $X$ such that $X \simeq X^{*}$ and $\mathrm{FPdim} X=2$. Then we have

(i) $\mathcal{C}$ is not modular.

Assume $\mathcal{C}=\mathcal{C}_{\text {ad }}$. Then we have in addition

(ii) There is a group isomorphism $G(\mathcal{C}) \simeq \mathbb{Z}_{2}$.

(iii) $G(\mathcal{C}) \subseteq \mathcal{C}^{\prime}$.

Proof. By [18, Theorem 4.2; Remark 4.4], $\mathcal{C}$ is Grothendieck equivalent to $\operatorname{Rep} D_{n}$ or Rep $\widetilde{D}_{2 n+1}$, for some $n \geq 1$. Since the universal grading group is a Grothendieck invariant, then in the first case $U(\mathcal{C})$ is isomorphic to $\mathbb{Z}_{2}$ if $n$ is even and is trivial if $n$ is odd. But $G(\mathcal{C})$, which is also a Grothendieck invariant, is isomorphic to $\mathbb{Z}_{2} \times \mathbb{Z}_{2}$ if $n$ is even and is isomorphic to $\mathbb{Z}_{2}$ if $n$ is odd, by Proposition 3.2 . Then $U(\mathcal{C})$ is not isomorphic to $\widehat{G(\mathcal{C})}$, for any $n$. Therefore $\mathcal{C}$ is not modular, by [11, Theorem 6.2]. Similarly, if $\mathcal{C}$ is 
Grothendieck equivalent to Rep $\widetilde{D}_{2 n+1}$, we have $U(\mathcal{C}) \simeq \mathbb{Z}_{2}$ and $G(\mathcal{C}) \simeq \mathbb{Z}_{4}$. Hence $\mathcal{C}$ is not modular in this case neither. This shows (i).

Notice that the assumption $\mathcal{C}=\mathcal{C}_{\text {ad }}$ implies that $\mathcal{C}$ is Grothendieck equivalent to $\operatorname{Rep} D_{n}$, for some $n$ odd. Then (ii) follows immediately from the fusion rules of Rep $D_{n}$, with $n$ odd (see Proposition 3.2). Since, by (i), $\mathcal{C}^{\prime}$ is not trivial, then $G\left(\mathcal{C}^{\prime}\right) \neq \mathbf{1}$, because c.d. $\left(\mathcal{C}^{\prime}\right) \subseteq\{1,2\}$ (c.f. Remark 4.3). By part (i), $G\left(\mathcal{C}^{\prime}\right)=G(\mathcal{C})$ and (iii) follows.

Remark 4.8. If $\mathcal{C}$ is a fusion category as in Lemma 4.7, then the assumption $\mathcal{C}=\mathcal{C}_{\text {ad }}$ is equivalent to saying that $\mathcal{C}$ is Grothendieck equivalent to $\operatorname{Rep} D_{n}$, for some $n \geq 1$ odd.

Lemma 4.9. Suppose that $\mathcal{C}=\mathcal{C}_{\mathrm{ad}}$. Then $\mathcal{C}$ is generated by fusion subcategories $\mathcal{D}_{1}, \ldots, \mathcal{D}_{s}, s \geq 1$, where $\mathcal{D}_{i}$ is Grothendieck equivalent to $\operatorname{Rep} D_{n_{i}}$ and $n_{i}$ is an odd natural number, for all $i=1, \ldots, s$.

Proof. Let $\mathcal{C}=\mathcal{C}\left[X_{1}, \ldots, X_{s}\right]$ for some simple objects $X_{1}, \ldots, X_{s}$. Let $\mathcal{D}_{i}=$ $\mathcal{C}\left[X_{i}\right]$ be the fusion subcategory generated by $X_{i}, i=1, \ldots, s$. By Lemma 4.2, $\left(\mathcal{D}_{1}\right)_{\text {ad }}, \ldots,\left(\mathcal{D}_{s}\right)_{\text {ad }}$ generate $\mathcal{C}$ as a fusion category. Hence, it is enough to consider only those simple objects $X_{i}$ whose Frobenius-Perron dimension equals 2 (otherwise, FPdim $X_{i}=1$ and $X_{i} \otimes X_{i}^{*} \simeq \mathbf{1}$ ).

Moreover, iterating the application of Lemma 4.2, we may further assume that $\left|G\left[X_{i}\right]\right|=2$, for all $i=1, \ldots, s$. Thus we have a decomposition $X_{i} \otimes$ $X_{i}^{*} \simeq \mathbf{1} \oplus g_{i} \oplus X_{i}^{\prime}$, where $G\left[X_{i}\right]=\left\{\mathbf{1}, g_{i}\right\}$ and $X_{i}^{\prime}$ is a self-dual simple object of Frobenius-Perron dimension 2. Since $X_{i} \otimes X_{i}^{*}$ generates $\left(\mathcal{D}_{i}\right)_{\text {ad }}$, the above reductions allow us to assume that $\mathcal{D}_{i}=\mathcal{C}\left[X_{i}\right]$ with $X_{i}$ simple objects of $\mathcal{C}$ such that $\mathrm{FPdim} X_{i}=2$ and $X_{i} \simeq X_{i}^{*}, \forall i=1, \ldots, s$.

We claim that we can choose the $X_{i}$ 's in such a way that $\left(\mathcal{D}_{i}\right)_{\text {ad }} \simeq \mathcal{D}_{i}$. By [18, Theorem 4.2; Remark 4.4], $\mathcal{D}_{i}$ is Grothendieck equivalent to $\operatorname{Rep} D_{n_{i}}$ or to Rep $\widetilde{D}_{2 n_{i}+1}$. Iterating the application of Lemma 4.2 and using Lemma 3.8. we obtain that $\mathcal{C}=\mathcal{C}\left[\mathcal{D}_{1}, \ldots, \mathcal{D}_{s}\right]$, with $\mathcal{D}_{j}$ a fusion subcategory of $\mathcal{C}$ Grothendieck equivalent to $\operatorname{Rep} D_{n_{j}}, n_{j}$ odd, for all $j=1, \ldots, s$, as we wanted.

4.2. Proof of Theorems $\mathbf{1 . 1}$ and $\mathbf{1 . 2}$, Let $\mathcal{C}$ be a weakly integral fusion category. It follows from [11, Theorem 3.10] that either $\mathcal{C}$ is integral, or $\mathcal{C}$ is a $\mathbb{Z}_{2}$-extension of a fusion subcategory $\mathcal{D}$. In particular, if $\mathcal{C}=\mathcal{C}_{\text {ad }}$, then $\mathcal{C}$ is necessarily integral.

Lemma 4.10. Let $\mathcal{C}$ be fusion category and let $X, X^{\prime}$ be simple objects of $\mathcal{C}$. Then the following are equivalent:

(i) The tensor product $X^{*} \otimes X^{\prime}$ is simple.

(ii) For every simple object $Y \neq 1$ of $\mathcal{C}$, either $m\left(Y, X \otimes X^{*}\right)=0$ or $m\left(Y, X^{\prime} \otimes X^{*}\right)=0$.

In particular, if $X^{*} \otimes X^{\prime}$ is not simple, then $\mathcal{C}[X]_{\mathrm{ad}} \cap \mathcal{C}\left[X^{\prime}\right]_{\mathrm{ad}}$ is not trivial.

Proof. The equivalence between (i) and (ii) is proved in [1, Lemma 6.1] in the case where $\mathcal{C}$ is the category of (co)representations of a semisimple Hopf 
algebra. Note that the proof loc. cit. works in this more general context as well.

Proof of Theorem 1.1. The proof is by induction on FPdim $\mathcal{C}$. As pointed out at the beginning of this subsection, if $\mathcal{C}$ is not integral, then it is a $\mathbb{Z}_{2}$ extension of a fusion subcategory $\mathcal{D}$. Since $\mathcal{D}$ also satisfies the assumptions of the theorem, then $\mathcal{D}$ is solvable, by induction. Hence $\mathcal{C}$ is solvable as well.

We may thus assume that $\mathcal{C}$ is integral. Therefore c.d. $(\mathcal{C})=\{1,2\}$ and the results of the previous subsection apply. By Lemma 4.5, we may assume that $\mathcal{C}=\mathcal{C}_{\text {ad }}$. Then it follows from Lemma 4.9 that $\mathcal{C}=\mathcal{C}\left[\mathcal{D}_{1}, \ldots, \mathcal{D}_{s}\right]$, with $\mathcal{D}_{j}$ Grothendieck equivalent to $\operatorname{Rep} D_{n_{j}}, n_{j}$ odd, $\forall j=1, \ldots, s$.

By Lemma 4.7. $G\left(\mathcal{D}_{j}\right)=\left\{\mathbf{1}, g_{j}\right\}, \forall j=1, \ldots, s$. We claim that $g_{i}=g_{j}$ $\forall 1 \leq i, j \leq s$. Indeed, let $\mathcal{D}_{j}=\mathcal{C}\left[X^{(j)}\right]$, where $X^{(j)}=X_{1}^{(j)}$ in the notation of Proposition 3.6. Then we have $\left(X^{(j)}\right)^{\otimes 2}=\mathbf{1} \oplus g_{j} \oplus X_{2}^{(j)}$. Fix $1 \leq i, j \leq s$. Since $\mathcal{C}$ has no simple objects of Frobenius-Perron dimension 4 then $g_{i}=g_{j}$ or $X_{2}^{(j)} \simeq X_{2}^{(i)}$, by Lemma 4.10. In the first case we are done. In the second case, we note that $\left\{1, g_{j}\right\}=G\left[X_{2}^{(j)}\right]=G\left[X_{2}^{(i)}\right]=\left\{1, g_{i}\right\}$. Then $g_{j}=g_{i}$, as claimed. Let $g=g_{j}=g_{i}$.

By Lemma4.7, $g \in \mathcal{D}_{i}^{\prime}$, for all $i=1, \ldots, s$. Since $\mathcal{D}_{i}, 1 \leq i \leq s$, generate $\mathcal{C}$ then $g \in \mathcal{C}^{\prime}$. It follows from Theorem 4.1 (ii) that $\mathcal{C}$ is the equivariantization by $\mathbb{Z}_{2}$ of a braided fusion category $\widetilde{\mathcal{C}}$. In particular, $F \operatorname{dim} \widetilde{\mathcal{C}}=\mathrm{FPdim} \mathcal{C} / 2$ and c. d. $(\widetilde{\mathcal{C}}) \subseteq\{1,2\}$, by [8, Proof of Proposition 6.2 (1)], [20, Lemma 7.2]. By inductive hypothesis, $\widetilde{\mathcal{C}}$ is solvable. Then $\mathcal{C}$, being the equivariantization of a solvable fusion category by a solvable group is itself solvable [8, Proposition 4.5 (i)].

Theorem 4.11. Let $\mathcal{C}$ be a weakly integral braided fusion category that FPdim $X \leq 2$ for all simple object $X$ of $\mathcal{C}$. Assume in addition that $\mathcal{C}=\mathcal{C}_{\text {ad }}$. Then $\mathcal{C}$ is tensor Morita equivalent to a pointed fusion category $\mathcal{C}\left(A \rtimes \mathbb{Z}_{2}, \tilde{\omega}\right)$, where $A$ is an abelian group endowed with an action of $\mathbb{Z}_{2}$ by group automorphisms, and $\tilde{\omega}$ is a certain 3 -cocycle on the semidirect product $A \rtimes \mathbb{Z}_{2}$.

Proof. The assumption $\mathcal{C}=\mathcal{C}_{\text {ad }}$ implies that $\mathcal{C}$ is integral. Hence we may assume that c.d. $(\mathcal{C})=\{1,2\}$. By Lemma 4.9, $\mathcal{C}$ is generated by fusion subcategories $\mathcal{D}_{1}, \ldots, \mathcal{D}_{s}, s \geq 1$, where $\mathcal{D}_{i}$ is Grothendieck equivalent to $\operatorname{Rep} D_{n_{i}}$ and $n_{i}$ is an odd natural number, for all $i=1, \ldots, s$. Furthermore, as in the proof of Theorem 1.1, the assumption that $\mathcal{C}=\mathcal{C}_{\text {ad }}$ implies that $G\left(\mathcal{D}_{i}\right)=\{\mathbf{1}, g\}$, for all $1 \leq i \leq s$, and $\mathcal{C}[g] \simeq \operatorname{Rep} \mathbb{Z}_{2}$ is a tannakian subcategory of the Müger center $\mathcal{C}^{\prime}$. So that $\mathcal{C} \simeq \tilde{\mathcal{C}}^{\mathbb{Z}_{2}}$ is an equivariantization of a braided fusion category $\tilde{\mathcal{C}}$.

Equivariantization under a group action gives rise to exact sequences of fusion categories [3, Subsection 5.3]. In our situation we have an exact sequence of braided tensor functors

$$
\operatorname{Rep} \mathbb{Z}_{2} \rightarrow \mathcal{C} \stackrel{F}{\rightarrow} \tilde{\mathcal{C}}
$$


In addition, since $\mathcal{C}[g] \subseteq \mathcal{D}_{i}$, then (4.3) induces by restriction an exact sequence

$$
\operatorname{Rep} \mathbb{Z}_{2} \rightarrow \mathcal{D}_{i} \rightarrow \tilde{\mathcal{C}}_{i}
$$

for all $i=1, \ldots, s$, where $\tilde{\mathcal{C}}_{i}$ is the essential image of $\mathcal{D}_{i}$ in $\tilde{\mathcal{C}}$ under the functor $F$. Hence $\tilde{\mathcal{C}}_{i}$ is a fusion subcategory of $\tilde{\mathcal{C}}$, for all $i$, and moreover $\tilde{\mathcal{C}}_{1}, \ldots, \tilde{\mathcal{C}}_{s}$ generate $\tilde{\mathcal{C}}$ as a fusion category. Note in addition that c. d. $(\tilde{\mathcal{C}})$, c. d. $\left(\tilde{\mathcal{C}}_{i}\right) \subseteq$ $\{1,2\}$, for all $i=1, \ldots, s$. On the other hand, exactness of the sequence (4.4) implies that $2 n_{i}=\mathrm{FPdim} \mathcal{D}_{i}=2 \mathrm{FP} \operatorname{dim} \tilde{\mathcal{C}}_{i}$ [3, Proposition 4.10]. Hence FPdim $\tilde{\mathcal{C}}_{i}=n_{i}$ is an odd natural number.

Since $\tilde{\mathcal{C}}_{i}$ is an integral braided fusion category, then the Frobenius-Perron dimension of every simple object of $\tilde{\mathcal{C}}_{i}$ divides the Frobenius-Perron dimension of $\tilde{\mathcal{C}}_{i}$ [8, Theorem 2.11]. Thus we get that $\operatorname{FPdim} Y=1$, for all $Y \in \operatorname{Irr}\left(\tilde{\mathcal{C}}_{i}\right)$. That is, $\tilde{\mathcal{C}}_{i}$ is a pointed braided fusion category, for all $i=1, \ldots, s$. Since $\tilde{\mathcal{C}}_{1}, \ldots, \tilde{\mathcal{C}}_{s}$ generate $\tilde{\mathcal{C}}$ as a fusion category, then $\tilde{\mathcal{C}}$ is also pointed. Therefore $\tilde{\mathcal{C}} \simeq \mathcal{C}(A, \omega)$ as fusion categories, where $A$ is an abelian group and $\omega \in H^{3}\left(A, k^{\times}\right)$.

Group actions on pointed categories were classified by Tambara 23. In view of [23, Theorem 4.1] and [21, Proposition 3.2], the fusion category $\mathcal{C} \simeq \tilde{\mathcal{C}}^{\mathbb{Z}_{2}}$ is tensor Morita equivalent to a pointed category $\mathcal{C}\left(A \rtimes \mathbb{Z}_{2}, \tilde{\omega}\right)$, where the semidirect product $A \rtimes \mathbb{Z}_{2}$ is with respect of the induced action of $\mathbb{Z}_{2}$ on the group $A$ of invertible objects of $\tilde{\mathcal{C}}$, and $\tilde{\omega}$ is a certain 3 -cocycle on $A \rtimes \mathbb{Z}_{2}$.

Proof of Theorem 1.2. The proof is an immediate consequence of Theorem 4.11.

Remark 4.12. Let $\mathcal{C}$ be a braided fusion category such that c. d. $(\mathcal{C})=\{1,2\}$. Suppose that $\mathcal{C}$ is nilpotent. By [4, Theorem 1.1] $\mathcal{C}$ admits a unique decomposition (up to the order of factors) into a tensor product $\mathcal{C}_{1} \otimes \cdots \otimes \mathcal{C}_{m}$, where $\mathcal{C}_{i}$ are braided fusion categories of Frobenius-Perron dimension $p_{i}^{m_{i}}$, for some pairwise distinct prime numbers $p_{1}, \ldots, p_{m}$. Then $\mathcal{C}_{i}$ is an integral braided fusion category, for all $i=1, \ldots, m$, and by [8, Theorem 2.11], we get that $\mathcal{C}_{i}$ is pointed whenever $p_{i}>2$. Hence $\mathcal{C} \simeq \mathcal{C}_{1} \otimes \mathcal{B}$ as braided fusion categories, where $\mathcal{C}_{1}$ is a braided fusion category of Frobenius-Perron dimension $2^{m}$ such that c. d. $\left(\mathcal{C}_{1}\right)=\{1,2\}$, and $\mathcal{B}$ is a pointed braided fusion category.

\section{REFERENCES}

[1] J. Bichon and S. NAtale, Hopf algebra deformations of binary polyhedral groups, Transf. Groups 16, 339-374 (2011).

[2] A. Bruguières, Catégories prémodulaires, modularisations et invariants des variétés de dimension 3, Math. Ann. 316, 215-236 (2000).

[3] A. Bruguières and S. Natale, Exact sequences of tensor categories, Int. Math. Res. Not. 2011 (24), 5644-5705 (2011).

[4] V. Drinfeld, S. Gelaki, D. Nikshych and V. Ostrik, Group-theoretical properties of nilpotent modular categories, preprint arXiv:0704.0195 (2007). 
[5] V. Drinfeld, S. Gelaki, D. Nikshych and V. Ostrik, On braided fusion categories I, Sel. Math. New Ser. 16, 1-119 (2010).

[6] P. Etingof, S. Gelaki and V. Ostrik, Classification of fusion categories of dimension pq, Int. Math. Res. Not. 2004:57, 3041-3056 (2004).

[7] P. Etingof, D. Nikshych and V. Ostrik, On fusion categories, Ann. Math. 162, 581-642 (2005).

[8] P. Etingof, D. Nikshych and V. Ostrik, Weakly group-theoretical and solvable fusion categories, Adv. Math. 226, 176-205 (2011).

[9] P. Etingof, E. Rowell and S. Witherspoon, Braid group representations from quantum doubles of finite groups, Pacific J. Math. 234, 33-42 (2008).

[10] S. Gelaki, D. NAIDU and D. NiKshyCh, Centers of graded fusion categories, Algebra Number Theory 3, 959-990 (2009).

[11] S. GELAKI and D. Nikshych, Nilpotent fusion categories, Adv. Math. 217, 1053-1071 (2008)

[12] I. IsAACS, Character theory of finite groups, Pure and Applied Mathematics 69, Academic Press, New York, (1976).

[13] J. Liptrap, Generalized Tambara-Yamagami categories, to appear in J. Algebra, preprint arXiv:1002.3166v2 (2010).

[14] A. MASUOKA, Cocycle deformations and Galois objects for some cosemisimple Hopf algebras of finite dimension, Contemp. Math. 267, 195-214 (2000).

[15] M. MÜGER, Galois theory for braided tensor categories and the modular closure, Adv. Math. 150, 151-201 (2000).

[16] M. MÜGER, On the structure of modular categories, Proc. Lond. Math. Soc. 3, 87, 291-308 (2003).

[17] D. Naidu, D. Nikshych and Sarah Witherspoon, Fusion subcategories of representation categories of twisted quantum doubles of finite groups, Int. Math. Res. Not. 2009 (22) 4183-4219 (2009).

[18] D. NAIDU and E. Rowell, A finiteness property for braided fusion categories, preprint arXiv:0903.4157v4.

[19] S. NATALE, On group-theoretical Hopf algebras and exact factorizations of finite groups, J. Algebra 270, 199-211 (2003).

[20] S. Natale and J. Plavnik, On fusion categories with few irreducibles degrees, to appear in Algebra Number Theory, preprint arXiv:1103.2340v2.

[21] D. NikShyCH, Non group-theoretical semisimple Hopf algebras from group actions on fusion categories, Sel. Math. 14, 145-161 (2008).

[22] J. SiEHLER, Braided near-group categories, preprint arXiv:math/0011037v1.

[23] D. TAMBARA, Invariants and semi-direct products for finite group actions on tensor categories, J. Math. Soc. Japan 53, 429-456 (2001).

[24] D. TAMBARA and S. YAMAGAmi, Tensor categories with fusion rules of self-duality for finite abelian groups, J. Algebra 209, 692-707 (1998).

[25] J. Thornton, On braided near-group categories, preprint arXiv:1102.4640v1

Facultad de Matemática, Astronomía y Física, Universidad Nacional de Córdoba, CiEM - CONiCET, (5000) Ciudad Universitaria, Córdoba, Argentina E-mail address: natale@famaf.unc.edu.ar, plavnik@famaf.unc.edu.ar

URL: http://www.famaf.unc.edu.ar/ natale 